Results Of the 15 patients, 18 eyes were treated with IAC. $46 \%$ had left-sided disease $(n=7), 33 \%$ had right-sided disease $(n=5)$, and $20 \%$ had bilateral disease $(n=3)$. The majority were male $(n=10)$. Average age at the start of treatment was 1 year 5.5 months. A total of 66 treatments were performed, with a median of 4 per patient or 3.5 per involved eye. Of the 18 eyes treated, 12 (66\%) initially had anterograde ophthalmic artery flow. Drug delivery was accomplished using direct ophthalmic catheterization $(n=35)$, ICA balloon technique $(n=18)$, ECA balloon technique $(n=7)$, and via ECA branch catheterization $(n=6)$. A flow reversal event was observed a total of 5 times (28\% of eyes), each necessitating a change in drug delivery technique. All 5 events were in patients receiving multi-agent chemotherapy. These events occurred in 4 patients and involved 5 separate eyes, never more than once per eye. Flow reversal events were seen in both eyes $(l e f t=3$, right $=2)$. On average, events occurred between the third and fourth treatment (range 2 to 6). No correlation was found between reversal event and treatment technique.

Conclusions Ophthalmic artery flow is variable in RB patients treated with IAC. Further, mid-treatment shifts between anterograde and retrograde ophthalmic artery filling are common and necessitate variation in delivery methods throughout a single treatment course. In our analysis all flow reversal events were associated with multi-agent chemotherapy (as opposed to Melphalan alone). Although the correlation was not statistically significant,our analysis is limited by power. Future investigation is necessary to elucidate the nature of these variations, such as if they are a direct response to the number of chemotherapy agents utilized. Regardless, interventionalists should be comfortable and prepared to use various techniques independent of a given patient's treatment history.

Disclosures M. Feldman: None. H. Grimaudo: None. S. Roth: None. H. Vance: None. A. Daniels: None. M. Froehler: 1; C; Genentech, Medtronic, Stryker, Microvention, and Penumbra. 2; C; Genentech, Medtronic, Stryker, Balt USA, Viz.ai, and Corindus.

\section{P-029 IMPACT OF IN-HOSPITAL DELIRIUM ON OUTCOMES OF ACUTE ISCHEMIC STROKE}

${ }^{1} \mathrm{D}$ Pujara*, ${ }^{2} \mathrm{H}$ Kamal, ${ }^{3} \mathrm{O}$ Mir, ${ }^{4} \mathrm{~S}$ Reddy, ${ }^{5} \mathrm{~K}$ Parsha, ${ }^{6} \mathrm{~S}$ Patil, ${ }^{7} \mathrm{~B}$ Gogia, ${ }^{7} \mathrm{P}$ Rai, ${ }^{8} \mathrm{C}$ Sitton, ${ }^{9} \mathrm{M}$ Chen, ${ }^{10} \mathrm{M}$ Abraham, ${ }^{11} \mathrm{M}$ Hussain, ${ }^{12} \mathrm{~A}$ Hassan, ${ }^{1} \mathrm{~A}$ Sarraj. ${ }^{1}$ Neurology, McGovern Medical School, Houston, TX; ${ }^{2}$ Neurology, Westchester Medical Center, New York, NY; ${ }^{3}$ Neurology, New York University, New York, NY; ${ }^{4}$ Neurology, Mercy Hospital, Fort Smith, $A R ;{ }^{5}$ Neurology, Semmes murphy clinic, Memphis, TN; ${ }^{6}$ Internal Medicine, Northeast Internal Medicine Associates, Fort Wayne, IN; ${ }^{7}$ Neurology, UT Medical Branch, Galveston, $T X_{i}{ }^{8}$ Neuroradiology, McGovern Medical School, Houston, TX; ${ }^{9}$ Neurology, Rush University Medical Center, Chicago, IL; ${ }^{10}$ Neurology, Kansas University Medical Center, Kansas City, KS; ${ }^{11}$ Neurology, Cleveland Clinic, Cleveland, TX; ${ }^{12}$ Neurology, UT Rio Grande Valley, Harlingen, $T X$

\subsection{6/neurintsurg-2021-SNIS.65}

Introduction Delirium is a common complication after acute ischemic stroke(AIS) and is usually associated with worse outcomes. Nationally representative estimates on the prevalence of in-hospital delirium after AIS are not well established. Similarly, how delirium affects the clinical outcomes and hospitalization costs is also unexplored.

Methods We reviewed Nationwide Inpatient Sample(NIS), a database containing 20\% inpatient admissions across the United States between 2012-2014. Standardized ICD-9-CM codes were used to identify incidence of delirium, its association with reperfusion therapy(IV-tPA and endovascular thrombectomy(EVT)) and outcomes including in-hospital mortality, discharge disposition, length of stay(LOS) and hospitalization costs in patients presenting with AIS, overall and in a propensity-matched cohort.

Results Of 356,952 cases with AIS between 2012-2014, delirium was present in 12,412 (3.5\%). Patients who exhibited delirium were older (non-delirium (ND):72 (60-82) years vs delirium (D):76 (65-85) years, $\mathrm{p}<0.001)$ and delirium was more common in females (ND:176,267 (51.2\%) vs D:6,748 (54.4\%), $\quad \mathrm{p}<0.001)$, caucasians $\quad(\mathrm{ND}: 229,094 \quad(70.1 \%) \quad$ vs $\mathrm{D}: 8,532(72.9 \%), \mathrm{p}<0.001)$ and patients with Medicare as the primary payer (ND:226,351 (65.8\%) vs D:9250 (74.7\%), $\mathrm{p}<0.001)$. These patients also exhibited more frequent history of congestive heart failure (ND:50,665 (14.7\%) vs D:2189 $(17.6 \%), \mathrm{p}<0.001)$, hypothyroidism (ND:47446 (13.8\%) vs D:1901 (15.3\%),p<0.001), renal failure (ND:54,388 (15.8\%) vs $\mathrm{D}: 2313(18.6 \%), \mathrm{p}<0.001)$ and concurrent fluid and electrolyte disorders (ND:90,242 (26.2\%) vs D:4989 (40.2\%), $\mathrm{p}<0.001)$.

These patients demonstrated history of neuropsychiatric conditions including depression (ND:36,270 (10.5\%) vs $\mathrm{D}: 1,638$ (13.2\%), $\mathrm{p}<0.001)$, psychosis (ND:12,559 (3.6\%) vs $\mathrm{D}: 883$ (7.1\%), $\mathrm{p}<0.001)$, alcohol abuse (ND:15,498 (4.5\%) vs $\mathrm{D}: 633$ (5.1\%), $\mathrm{p}<0.001)$ and other neurological diseases (ND:16,638 (4.8\%) vs $\mathrm{D}: 2,514 \quad(20.3 \%), \quad \mathrm{p}<0.001)$ more frequently.

Overall, the prevalence of delirium was lower in patients receiving reperfusion therapy (No reperfusion:11870/321805 (3.6\%) vs Only IV-tPA:435/19079 (2.23\%) vs only EVT:64/ 1999 (3.1\%) vs tPA + EVT:43/1657 (2.5\%),P<0.001).

Older age (aOR 1.02,95\%CI:1.01-1.02,p<0.001), history of alcohol abuse (aOR:1.25,95\%CI:1.15-1.37,p<0.001), history of depression (aOR:1.18,95\%CI:1.11-1.24,p<0.001), psychosis (aOR:1.88,95\%CI:1.75-2.03,p<0.001) and other neurological diseases (aOR:4.45,95\%CI:4.23-4.67,p<0.001) were significant predictors of developing in-hospital delirium after acute ischemic stroke.

Delirium was associated with higher in-hospital mortality (D:1111(9.0\%) vs ND:23203(6.7\%), p<0.001), increased length of in-hospital stay (D:6(3-10) vs ND:4(2-7) days, $\mathrm{p}<0.001)$ and total charges associated with inpatient admissions (D: \$39423 (21513-83927) vs \$34087 (19970-64254), $\mathrm{p}<0.001)$. After adjusting for baseline patient characteristics including demographics and comorbidities, hospital characteristics and utilization of IV tPA and EVT, delirium was associated with an $8 \%$ increase in the risk of in-hospital deaths (aOR:1.08, 95\%CI:1.01-1.15, $\mathrm{p}=0.024)$, an increase of 1.4 days in hospital LOS (adj. $\beta: 1.39,95 \% \mathrm{CI}: 1.23-1.54, \mathrm{p}<0.001$ ), $\sim \$ 8734$ increase in total hospital charges (adj. $\beta: 8734,95 \%$ CI:6764-10705, p <0.001) and 31\% decrease in odds of home discharge (aOR:0.69,95\%CI0.67-0.72,p<0.001). The results were confirmed in the propensity matched cohort of 12,107 matched pairs of patients with and without delirium (in-hospital death-aOR:1.10,95\%CI:1.01-1.20,p=0.044; LOS-adj. $\beta: 1.11,95 \% \mathrm{CI}: 0.86-1.36, \mathrm{p}<0.001 ;$ total charges-adj. $\beta$ : $\$ 5914,95 \% \mathrm{CI}: 2623-9206, \mathrm{p}<0.001$ and home discharge-adj. OR:0.74, 95\%CI:0.70-0.78,p<0.001).

Conclusions Delirium complicates $\sim 3.5 \%$ of AIS admissions, and is associated with increased risk of in-hospital death, longer stay, higher charges and decrease in likelihood of home discharge. 
Disclosures D. Pujara: None. H. Kamal: None. O. Mir: None. S. Reddy: None. K. Parsha: None. S. Patil: None. B. Gogia: None. P. Rai: None. C. Sitton: None. M. Chen: None. M. Abraham: None. M. Hussain: None. A. Hassan: None. A. Sarraj: None.

\section{P-030 VOXEL-BASED CALCULATIONS OF INTRASACCULAR ANEURYSM AND DEVICE VOLUME FILL}

S Schwartz*, B Fennell, C Settanni, N Norris, T Becker. Bioengineering Program, Mechanical Engineering, Northern Arizona Univeristy, Flagstaff, $A Z$

10.1136/neurintsurg-2021-SNIS.66

Introduction Various embolization techniques are available for intracranial aneurysms. The precise volume measurement of an aneurysm sac can help improve embolization techniques, such as: coils, liquid embolics, flow disruptors, and flow diverters. Aneurysm sizing and volumetric information help interventionalists assess flow and stability pre-treatment and potential remnant or recanalization risks post-treatment.

Materials and Methods This research project applies voxelbased volume calculations, from CT or MRI medical imaging data, to determine accurate 3-D aneurysm volume calculations. Additionally, the application can display clinically relevant parameters, such as aneurysm neck diameter, dome height and midline-dome width (for dome:neck (D:N) ratio calculations). To develop the measurement techniques, wide-neck canine aneurysm models $(n=10)$ were scanned with a Siemens Inveon Micro-CT scanner (University of Arizona TBIR, Tucson, AZ) and analyzed with InVesalius 3.0 software (Ministry of Health, Brazil).

The software loads the imaging data, discretizes the intrasaccular aneurysm, separating the device volume from the aneurysm sac. This data was compared to physical measurements of excised aneurysms and calibrated 2-D angiographic images.

Results Invesalius image processing techniques helped determine the domain of aneurysms with less than $5 \%$ volumetric error, when compared to measurements recorded during the aneurysm creation surgery. Current measurement techniques in 2-D planes have errors as high as 30\%. This technique helps determine the macroscopic properties of aneurysms, as well as the volumes of abnormal aneurysm shapes. The software was also used to measure $\%$ fill of a liquid embolic (NeuroCURE) delivered to the 10 canine aneurysms. In all 10 cases the precise delivery of NeuroCURE resulted in 90$100 \%$ aneurysm sac filling, versus $<30 \%$ for traditional coiling techniques.

Conclusion As new aneurysm treatment devices are developed and greater\% fill of the aneurysm sac is attempted, the need for precise aneurysm volume calculations will be of high priority. This project brings together clinical and engineering expertise to translate medical imaging data directly into volumetric measurements with highly precise calculations that are currently not available from 2-D angiographic images. Measuring the size and dimensional properties of aneurysms with voxel-based volume calculations provides a fast, reliable and repeatable resource for aneurysm assessment. This technique will help interventionalists appropriately assess risk and treatment options for a broad range of aneurysm morphologies.
Disclosures S. Schwartz: 1; C; 5R42NS097069-03 (NIH Grant\#)). B. Fennell: 1; C; 5R42NS097069-03 (NIH Grant \#). C. Settanni: 1; C; 5R42NS097069-03 (NIH Grant \#). N. Norris: 1; C; 5R42NS097069-03 (NIH Grant \#). T. Becker: 1; C; 5R42NS097069-03 (NIH Grant \#).

\section{P-031 EFFECT OF COVID-19 ON ACUTE ISCHEMIC STROKE: POPULATION-LEVEL EXPERIENCE}

Y Kim*, S Khose, S Salazar-Marioni, R Abdelkhaleq, S Sheth. Department of Neurology, UTHealth MGovern Medical School, Houston, TX

10.1136/neurintsurg-2021-SNIS.67

Introduction Several studies report substantial decreases in the volume of acute ischemic stroke (AIS) during the early stage of coronavirus disease 2019 (COVID-19) pandemic. Differences in characteristics of strokes such as increasing rates of large vessel occlusion (LVO) stroke were also observed comparing between pre-COVID and COVID eras. However, these findings were often based on data from selected hospitals or networks and estimates may be biased by level of stroke care and baseline case mix. A population-based study including rural hospitals and non-stroke centers covering a longer observation period beyond the early-pandemic epoch may lead to more accurate insights.

Methods We conducted a retrospective population-based study using the Texas Inpatient Public Use Data File, capturing all discharges from hospitals in the State of Texas excluding federal hospitals. We assessed quarterly changes in volume, characteristics, care of AIS, and patient outcomes from April 1, 2019-June 30, 2020 and compared two time periods. 2020Q2 was defined as the pandemic period and the previous one year as the pre-pandemic period. Cases were defined as inpatient hospitalizations to acute care hospitals with a primary diagnosis of AIS. ICD-10 diagnosis and procedure codes were used to identify AIS, LVO, use of intravenous tissue plasminogen activator (IV-tPA) and endovascular treatment (EVT). Hospitals were determined as CSC (Comprehensive Stroke Center)

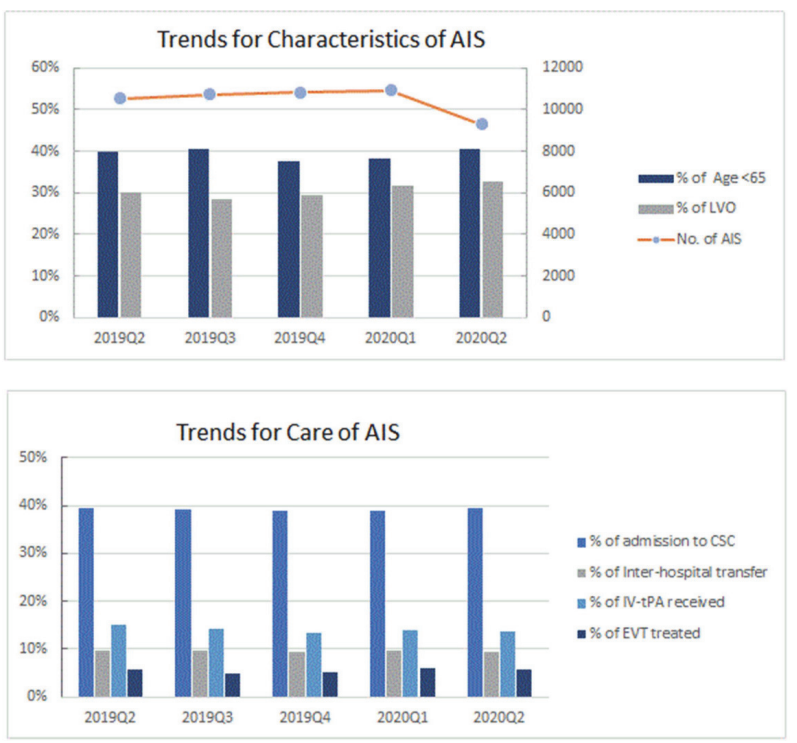

Abstact P-031 Figure 1 